\title{
Experiment on marine floating start of ESGM
}

\author{
LIU zhi, ZHOU jian, LIU hang, YU shang-yong \\ China Satellite Maritime Tracking and Control Department, Jiangyin, 214431, China
}

Key words: electrostatic gyroscope monitor; starting mode; six-time calibration; gyro calibration

\begin{abstract}
The start of electrostatic gyro monitor(ESGM)requires sailing at uniform velocity and constant latitude .This is difficult to achieve and hard for the sailing . Analyzing the data of floating start base on the starting principle of ESGM .The result shows that, ESGM can use floating startmode in some conditions.
\end{abstract}

\section{Introduction}

In the measurement of the ship in the distant sea, you need to use the electrostatic gyro monitor (hereinafter referred to as ESGM) output of high precision, high stability and attitude data. ESGM features ESGM and platform inertial navigation equipment (referred to as INS) with the use of real-time monitoring of INS navigation error. In the case where the optical theodolite can not be measured, the accuracy of the INS output heading can be increased to provide for the measurement of the measurement equipment on the ship.

\section{ESGM boot process}

The accuracy of the ESGM system depends on the estimated accuracy of the parameters used by the channel during the start-up process. The estimation of these parameters has a direct relationship with the start-up phase of the entire ESGM, which includes gyro alignment, gyro start, six calibration, 48 hour calibration and so on.

\subsection{Gyro start}

The two electrostatic gyro in the alignment phase, has been roughly geometric symmetry axis, respectively, according to the requirements of the Earth polar axis and parallel to the equatorial plane. As the rotor has not yet rotated, and can not constitute the inertial space. Therefore, it is necessary to accelerate the upper and lower gyroscope to rotate it in order to establish the inertial space. The acceleration process is divided into three stages: acceleration, damping and secondary acceleration. Eventually turning the gyro speed to $300 \mathrm{~Hz}$. Then to enter the follow-up state, the initial formation of inertial coordinate system.

\subsection{Six times of school}

Six calibrations is to establish the solution channel, and the initial alignment process, the need to calculate six times, it is called six schools.

The specific process is to establish the solution channel to calculate the instantaneous local time angle, the declination of the gyroscope according to the initial gyro drift parameters, the Earth's rotation angular rate, the gyro momentum moment axis relative to the equatorial coordinate system, And the corner of Greenwich, that is: 


$$
\left\{\begin{array}{l}
S_{i}^{P}(t)=S_{i}(0)+\Delta S_{i}^{\omega_{e}}(t)+\Delta S_{i}^{C}(t) \\
\delta_{i}^{P}(t)=\delta_{i}(0)+\Delta \delta_{i}^{\omega_{e}}(t)+\Delta \delta_{i}^{C}(t) \\
S_{i}^{* P}(t)=S_{i}^{P}(t)+\lambda(t)
\end{array}\right.
$$

The relationship between the position angle of the gyro momentum moment axis in the equatorial coordinate system and its position angle in the horizontal coordinate system is:

$$
\left\{\begin{array}{l}
\cos h_{i} \sin A_{i}=-\cos \delta_{i} \sin S_{i}^{*} \\
\sin h_{i}=\sin \delta_{i} \sin \varphi+\cos \delta_{i} \cos \varphi \cos S_{i}^{*} \\
\cos h_{i} \cos A_{i}=\sin \delta_{i} \cos \varphi-\cos \delta_{i} \sin \varphi \cos S_{i}^{*} \\
S_{i}=S_{i}^{*}-\lambda
\end{array}\right.
$$

\subsection{Calibration stage}

After the ESGM completes the six calibrations, the parameters needed for the channel are further accurately solved, including the process of the initial position angle and the gyro drift parameters of the gyroscope, that is, the process of calculating the instantaneous solution value of the channel after the transshipment operation is accurately calculated.

The calibration process is to estimate the initial position error and the gyro drift parameter error by using the best filtering method, and then compensate the corresponding value of the solution channel. Calibration process schematic diagram shown in Figure 1.

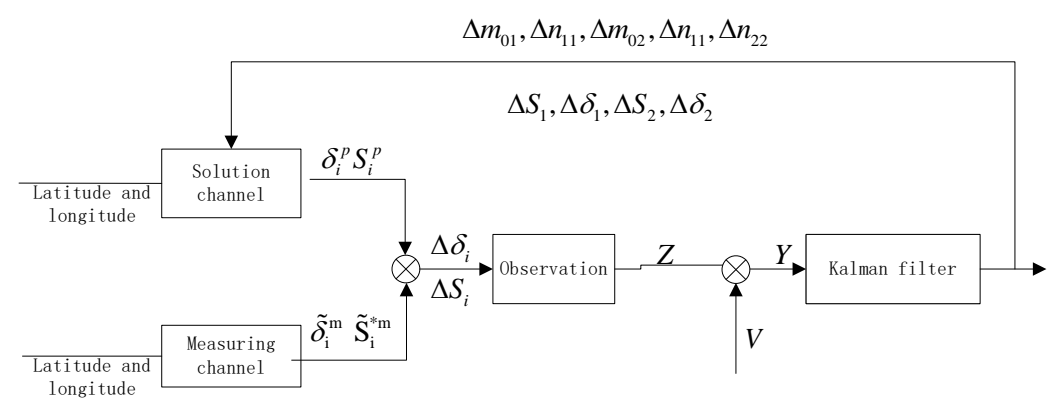

Figure 1. Electrostatic calibration process schematic

\section{Drift start analysis}

2.1. Start the same latitude

ESGM sea generally use the same latitude under the mode of landing, start the process complex.

Sea and other latitude start, but also need a number of security conditions:

A). There are continuous outside position and speed information: generally provided by satellite navigation equipment.

B). Because the ESGM can not work independently, it requires the INS device to provide support: typically 24 hours after the INS runs, the INS can be used as a support when it is stable.

C). From the start of the ESGM to the 7th hour: the survey ship sails at a certain speed for equal-length straight-line.

D). From ESGM start 7 hours to 60 hours between: measuring the ship as far as possible to maintain uniform navigation, such as the need to turn, then require heading, speed fine-tuning.

E). Other time: measuring ship can be mobile (steering or speed), but the mobility should not be frequent.

Through the previous analysis, in the ESGM system, the change of the gyro height angle corresponds to the latitude change and the azimuth change corresponds to the heading change. So 
under normal circumstances are usually used to determine the latitude of the latitude and the amount of change in the amount of change. And in the gyroscope alignment and start-up phase, the gyro height angle and the azimuth angle need to be adjusted in order to ensure six calibration and system calibration process for gyro drift parameters and gyro initial position of the calculation accuracy.

\subsection{Drift start}

As part of the sea can not guarantee adequate and other latitude sailing, so the use of drift start test. Drifting start is generally carried out in the case of good sea conditions, at this time far from the ocean flow and flow rate is more stable, the carrier's heading and location are not fixed but the change is relatively small.

\section{A). Ship speed and latitude changes}

Through the previous analysis, in the ESGM system, the change of the gyro height angle corresponds to the latitude change and the azimuth change corresponds to the heading change. So under normal circumstances are usually used to determine the latitude of the latitude and the amount of change in the amount of change. And in the gyroscope alignment and start-up phase, the gyro height angle and the azimuth angle need to be adjusted in order to ensure six calibration and system calibration process for gyro drift parameters and gyro initial position of the calculation accuracy.
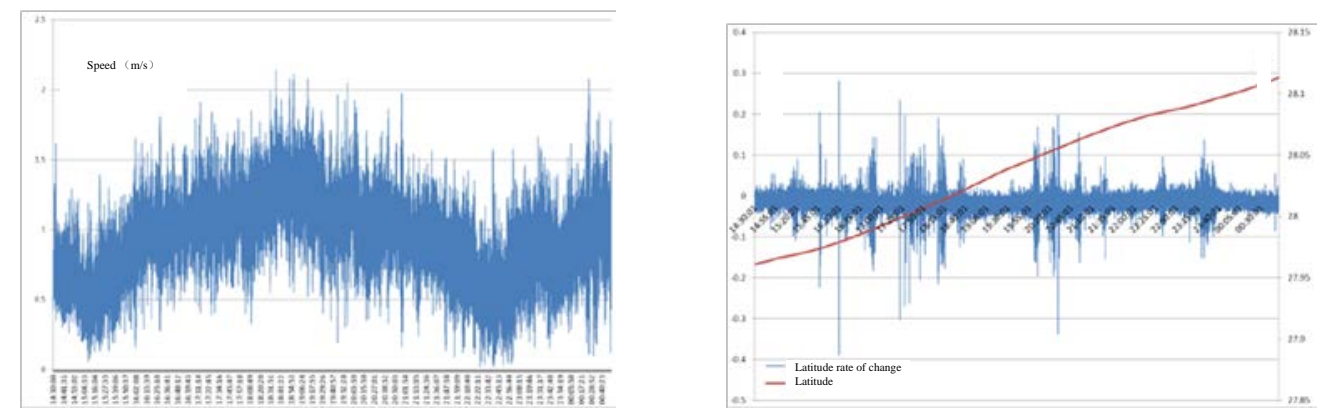

Figure 2. Drift in the ship speed Figure 3. The rate of change of the ship 's latitude in the start of drift.

Drift in the start, only the impact of ocean currents caused by the speed of the ship, and the speed is very small. Figure 3 shows the speed curve shows that drift in the start of the speed of $2 \mathrm{~m} / \mathrm{s}$ or less. Figure 4 shows the latitude and latitude rate of change within 10 hours after the start of the ESGM. Latitude basically no change, within 10 hours only change about $0.1^{\circ}$; latitude change rate is small, only within 0.3 ". In the drift, the ship only by the impact of ocean currents, speed, latitude and latitude change rate is small. Making the ESGM in the starting gyroscope height angle change is small, to ensure that the acceleration and calibration of the gyro stability.

B). Heading and heading rate of change

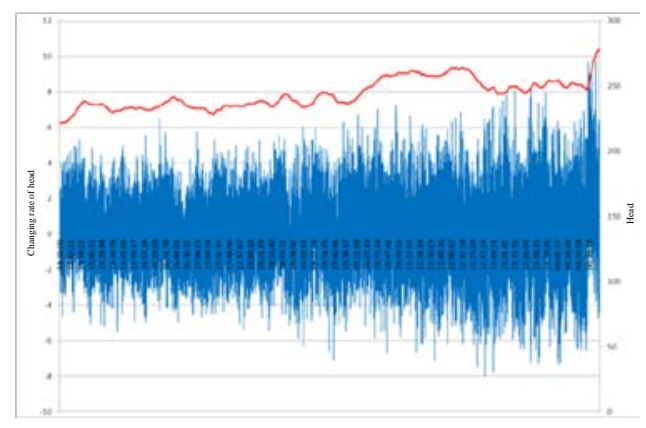

Figure 4. Heading rate of change and heading in drift start

Observe the curve of heading and heading change in Figure 4. ESGM drift in the start, the course 
of stability, from the start 10 hours, the course of change is stable, the rate of change within 10 cents. In the test, the heading changes smoothly, or basically no change.

\section{Post-navigation data analysis}

After the drift is started, the error of ESGM is recorded. And compared with the latitude sailing start to evaluate the accuracy of ESGM data after the drift start.

A). Position error

Figure 7 and Figure 8, respectively, is the same latitude heading start ESGM and drift after the navigation, ESGM position difference.

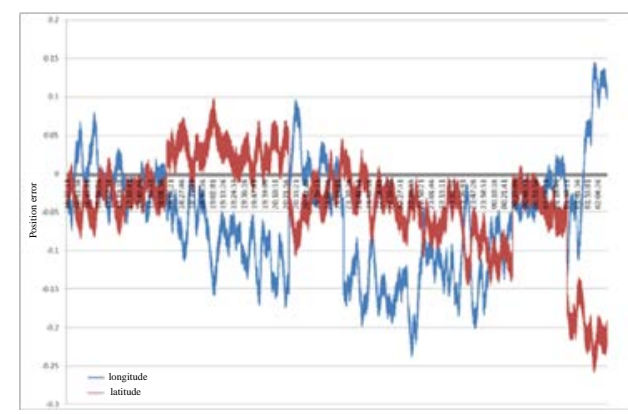

Figure 5. Isometric latency after starting latitude

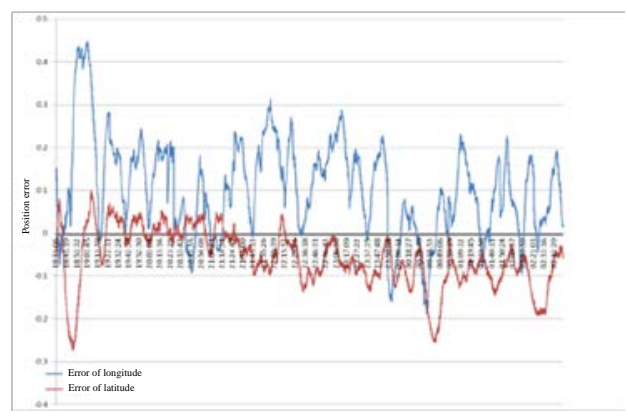

Figure 6. Air drift after drift start navigation

As can be seen from the graph of Fig. 7, the ESGM position difference is around 0.1 '. Figure 8 ESGM position accuracy of about 0.4 '.

B). Zero situation

The ESGM's zero position can be used to correct the operation of the ESGM after navigation.

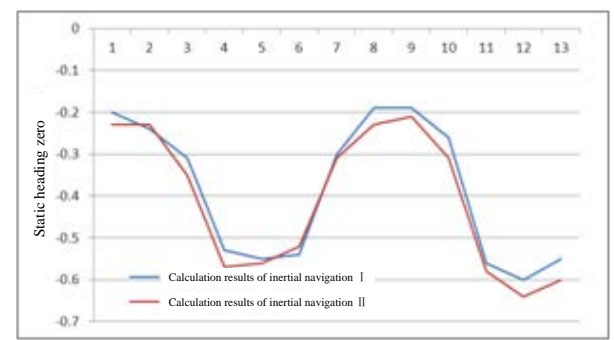

Figure 7. The heading zero after latitude start

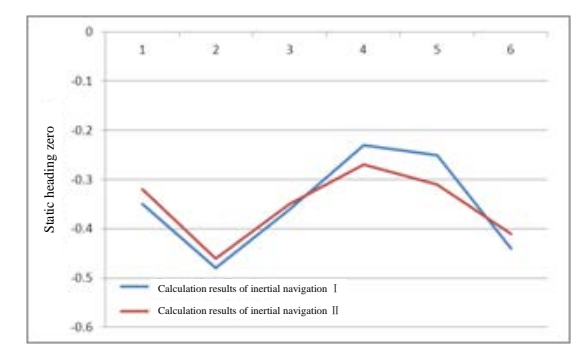

Figure 8. The heading zero position after drift

Figure 7 and Figure 8 compared to the curve, drift after the start of static zero stability, and after the same latitude sailing after the zero consistency is better.

\section{Summary}

A). Through the analysis and analysis of the key stages of the ESGM start-up, ESGM can be used to start the drift in the remote sea conditions. The ship's position mainly includes: the ship's course is stable and the change is stable, the change rate of the heading is less than $15^{\circ} / \mathrm{min}$; the ship's position (mainly refers to the latitude change) is relatively small or basically no change; the ship's horizontal and vertical pitch is less than $3^{\circ}$, Violent shaking.

B). The ship does not need power support during the drift. So taking into account the fuel consumption and ship engine wear and tear, etc. can be considered drift start. If the navigation conditions are limited, do not meet the latitude sailing start, you can also choose a better sea 
conditions with drift jet start.

C). Comparison of position accuracy can be seen, such as latitude after the start of the position accuracy is better. Taking into account the uncertainty of the sea conditions, so the conditions permit, the latitude start more conducive to the stable operation of ESGM.

D). Drift start test to do a lot of successful tests, but for the determination of the ultimate guarantee conditions need to be further verified by the test, follow-up will also be carried out on this issue to test.

\section{Reference:}

[1] Pan Liang. Space measurement ship ship position measurement technology [M]. Beijing: National Defense Industry Press, 2009.

[2] Liu Xinming, Sun Xuecheng. "Three times school" method for electrostatic gyro monitor [J]. Chinese Journal of Inertial Technology, 2011,19 (4): 0399-04.

[3] Zhou Haiyuan, Pan Liang. Comparison of several startup modes of electrostatic gyro monitor and its application analysis. Chinese Journal of Inertial Technology, 2015,23 (4): 0467-05. 